\title{
Synthesis of Monodisperse CdS Nanorods Catalyzed by Au Nanoparticles
}

\author{
Hongwang Zhang ${ }^{1}$, Savas Delikanli ${ }^{2}$, Yueling Qin ${ }^{2}$, Shuli $\mathrm{He}^{2,3}$, Mark Swihart ${ }^{1}(\bowtie)$, and Hao Zeng ${ }^{2}(\bowtie)$ \\ ${ }^{1}$ Department of Chemical and Biological Engineering, University at Buffalo, SUNY, Buffalo, NY 14260, USA \\ ${ }^{2}$ Department of Physics, University at Buffalo, SUNY, Buffalo, NY 14260, USA \\ ${ }^{3}$ Department of Physics, Capital Normal University, Beijing 100048, China \\ Received: 9 July 2008/ Revised: 3 August 2008/Accepted: 25 August 2008 \\ CTsinghua Press and Springer-Verlag 2008. This article is published with open access at Springerlink.com
}

\begin{abstract}
Semiconductor nanocrystals (dots, rods, wires, etc.) exhibit a wide range of electrical and optical properties that differ from those of the corresponding bulk materials. These properties depend on both nanocrystal size and shape. Compared with nanodots, nanorods have an additional degree of freedom, the length or aspect ratio, and reduced symmetry, which leads to anisotropic properties. In this paper, we report the Au nanoparticlecatalyzed colloidal synthesis of monodisperse CdS nanorods. Based on systematic high resolution transmission electron microscopy studies, we propose a growth mechanism for these nanorods.
\end{abstract}

\section{KEYWORDS}

Nanorods, monodisperse, catalytic growth, solution phase synthesis

\section{Introduction}

Semiconductor nanocrystals (dots, rods, wires, etc.) exhibit a wide range of electrical and optical properties that differ from those of the corresponding bulk materials. These properties depend on both nanocrystal (NC) size and shape [1]. In the last decade, there has been increasing research activity on dimensionality control of NC properties, since the electrons interact differently in nanostructures having different dimensionality. One-dimensional (1D) systems are critical to the function and integration of nanoscale devices since these are the lowest dimensional structures that can be used for efficient transport of electrons and optical excitations [2]. Compared with zero-dimensional (0D) quantum dots, 1D nanostructures - such as nanorods and nanowires - have reduced symmetry and an additional degree of freedom, i.e., the length or aspect ratio. They are capable of exhibiting physical phenomena, such as linearly polarized light emission, that are not observed in spherical nanocrystals. Semiconductor nanorods and nanowires have been attracting great interest for applications in nanoelectronics, photonics, data processing, and biological and medical sensing.

To date, significant progress has been made in the fabrication of 1D nanostructures for a variety of materials, mainly semiconductors but also including metals and metal oxides. Several approaches are commonly used for the generation of 1D nanostructures, including surfactant controlled growth, growth with catalysts (solution-liquid-solid,

Address correspondence to Mark Swihart, swihart@eng.buffalo.edu; Hao Zeng, haozeng@buffalo.edu 
SLS and vapor-liquid-solid, VLS), growth by oriented attachment, templated growth and growth in the presence of an external field $[3,4]$. Of these methods, the first two are the most commonly used techniques for the synthesis of 1D nanostructures. Peng and Alivisatos first demonstrated growth of CdSe nanorods using a binary mixture of the surfactants hexylphosphonic acid and trioctylphosphine oxide [5]. The preferential adsorption of phosphonic acid groups onto certain facets of the nanocrystals facilitated the anisotropic growth along the c-axis and thus induced the anisotropic growth of CdSe nanocrystals along this particular axis [5]. They also synthesized CdTe nanorods and branched nanorods using similar methods $[3,6,7]$. In the SLS approach, the catalysts are usually colloidal metal nanoparticles that are either injected into the solution or formed by nucleation in situ. They are believed to stay in a molten or near-molten state during the reaction. The precursor atoms dissolve in the seeds and crystals grow at the metal surface. Some examples of this method include the earliest synthesis of a series of semiconductor nanowires by Buhro et al. [8, 9], the synthesis of InAs nanorods with Au NCs as the catalysts by Kan and Banin [10], the synthesis of straight or branched PbSe nanowires and nanorods $[11,12]$ and CdSe nanowires [13], and the preparation of Si nanowires using Au NCs as catalysts [14].

CdS is a II-VI semiconductor with a Bohr radius of $2.4 \mathrm{~nm}$ [15] and a direct band gap of $2.4 \mathrm{eV}$ [16]. It can be used in photocatalytic hydrogen production and electricity generation [17], in thin film transistors [18], in light-emitting diodes, and in other nonlinear optical devices $[19,20]$. CdS nanorods or nanowires can be prepared by several approaches, including laserassisted catalytic growth [21], thermal evaporation of CdS nanopowders [22], templated growth [13, 23] and solvothermal routes [17, 24]. Recently, Carbonne et al. synthesized CdS nanorods using CdSe NPs as seeds [25]. Here we report a straightforward Au-catalyzed synthesis of monodisperse CdS nanorods with highly controlled diameter and aspect ratio. At lower reaction temperatures, amorphous CdS quantum dots were produced. At higher reaction temperatures, crystalline CdS nanorods were obtained. In some earlier work, $\mathrm{Au}-\mathrm{CdS}$ nanostructures with different morphologies were synthesized [26-28]. These reports differ from the present work either in terms of the morphologies of the products, due to subtle differences in synthetic parameters such as reaction temperature and solvent $[26,28]$, or the order of precursor and seed introduction [27]. We found that a reaction temperature of $300{ }^{\circ} \mathrm{C}$ is critical for the formation of nanorods. The size of the Au seed nanoparticles has an important influence on the length and diameter of the nanorods. Systematic high resolution transmission electron microscopy (HRTEM) studies have been carried out in order to investigate the growth mechanism for these nanostructures.

\section{Experimental}

Synthesis of Au nanoparticles. Au nanoparticles were synthesized by the well-known two-phase method of Brust et al. [29] or by heating $\mathrm{HAuCl}_{4}$ in tetralin in the presence of oleylamine [30].

Synthesis of CdS nanorods. CdS nanorods were synthesized by anisotropic growth in a high temperature organic solution phase, using $\mathrm{Au}$ nanoparticles as catalyst. In a typical synthesis of the CdS nanorods, $0.5 \mathrm{mmol} \mathrm{CdO}$ and $1 \mathrm{~mL}$ oleic acid were mixed with $10 \mathrm{~mL}$ octadecene in a $125 \mathrm{~mL}$ four-neck flask. Under nitrogen flow, the system was heated to $200{ }^{\circ} \mathrm{C}$ until a Cd-oleate complex was formed in the solution (which became colorless). After cooling the mixture to $100^{\circ} \mathrm{C}$, either $3 \mathrm{~nm}$ or $10 \mathrm{~nm} \mathrm{Au}$ nanoparticles (corresponding to $0.05 \mathrm{mmol} \mathrm{HAuCl}_{4}$ ) in hexane were injected and the hexane was distilled out. A $2.5 \mathrm{~mL}$ aliquot of $0.1 \mathrm{~mol} / \mathrm{L}$ sulfur solution (sulfur in a $1: 4 \mathrm{v} / \mathrm{v}$ oleylamine to phenyl ether mixture) was then injected. The mixture was kept at $120{ }^{\circ} \mathrm{C}$ for 0.5 hours, and then heated to $300{ }^{\circ} \mathrm{C}$ and kept at that temperature for 3 hours. After cooling to room temperature, the mixture was treated with ethanol and centrifuged. The precipitate was redispersed in hexane.

Characterization. Absorption spectra were collected using a Shimadzu model 3101PC UV-visNIR scanning spectrophotometer over a wavelength range from 300 to $800 \mathrm{~nm}$. Emission spectra were obtained using a Fluorolog-3 spectrofluorometer. 
The size and morphology of the nanorods were characterized by a JEOL JEM-100CX transmission electron microscope (TEM) at an acceleration voltage of $80 \mathrm{kV}$. The crystal structure and orientation relationship between the CdS nanorods and $\mathrm{Au}$ nanoparticles were investigated by a JEOL 2010 high resolution transmission electron microscope (HRTEM) at an acceleration voltage of $200 \mathrm{kV}$. X-ray diffraction patterns were recorded using a wide-angle powder X-ray diffractometer (XRD, Siemens D500) with $\mathrm{Cu} \mathrm{K} \alpha$ radiation.

\section{Results and Discussion}

When the reaction was performed at a relatively low temperature $\left(120{ }^{\circ} \mathrm{C}\right)$, a thin shell of CdS material was found to be coated on the outside of the $\mathrm{Au}$ nanoparticle seeds (TEM images not shown here). Electron diffraction results suggest that the shell is amorphous. It can be assumed that the main reaction in the low temperature process is the adsorption of the precursors and the nucleation of amorphous CdS shells on the Au nanoparticles, since it is well known that sulfur bonds strongly to Au. The samples do not show any photoluminescence.

At a higher reaction temperature $\left(300{ }^{\circ} \mathrm{C}\right)$, crystalline nanorods were formed. Figure 1 shows a low magnification TEM image of CdS nanorods with $3 \mathrm{~nm}$ Au nanoparticles attached at one end. The darker region is the Au seed and the lighter area is CdS. The dimensions of the CdS nanorods are $7 \pm 1$ $\mathrm{nm}$ in diameter and $25 \pm 3 \mathrm{~nm}$ in length. The HRTEM

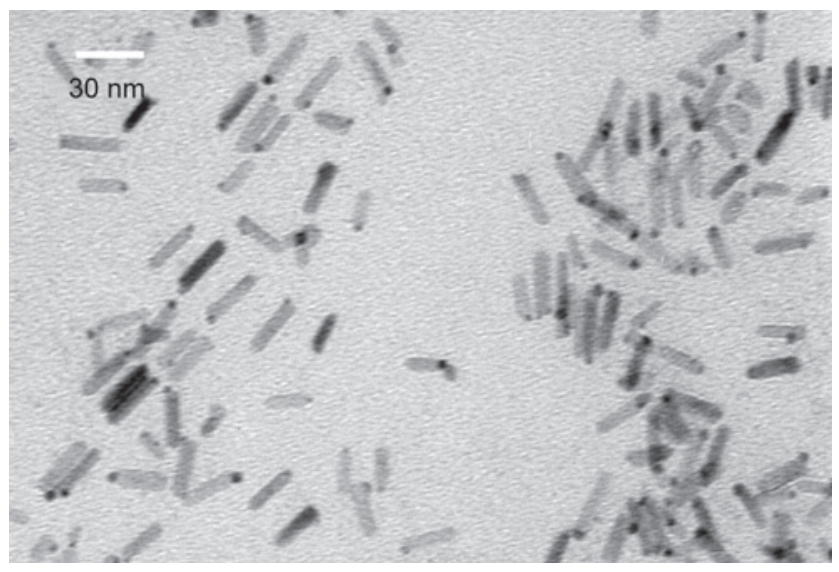

Figure 1 TEM image showing the $\mathrm{Au}-\mathrm{CdS}$ hybrid nanorods produced by the anisotropic growth of CdS rods, with diameter $7 \pm 1$ $\mathrm{nm}$ and length $25 \pm 3 \mathrm{~nm}$, on Au seeds (scale bar is $30 \mathrm{~nm}$ ) images of these binary hybrids are shown in Fig. 2 . Both the $\mathrm{Au}$ and CdS are well crystallized. Careful examination of many HRTEM images suggests that most CdS nanorods are single crystals. The nanorods have the wurzite structure, with about $95 \%$ of the rods grown with the c-axis oriented along the growth direction, as shown in Fig. 2(a). The inset is a fast Fourier transform (FFT) pattern, which clearly shows that the CdS nanorods are single crystals, and the pattern can be indexed to the [100] zone axis of the wurzite structure. A small fraction $(<5 \%)$ of the rods grow with their c-axis perpendicular to the long axis, as shown in Fig. 2(b).

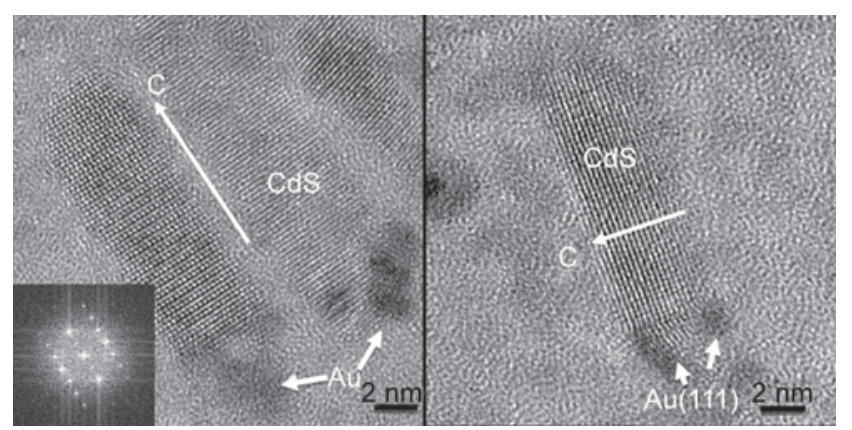

(a)

(b)

Figure 2 HRTEM images of the Au-CdS hybrid nanorods prepared by the anisotropic growth of CdS rods on $3 \mathrm{~nm}$ Au seeds. CdS rods can grow with their c-axis along (a) or perpendicular to (b) the growth direction. The inset in (a) is a fast Fourier transform (FFT) pattern of the rod on the right of the image. It can be indexed as [100] of wurzite CdS

When larger $(10 \mathrm{~nm}) \mathrm{Au}$ seeds were used as catalysts, the resulting nanorods are larger in dimensions, and monodisperse with uniform diameter and length. As shown in Fig. 3, the average diameter is $10 \pm 1 \mathrm{~nm}$ and length is $120 \pm 4 \mathrm{~nm}$. Most of the CdS nanorods are detached from the Au seeds and, locally, they self-assemble into aligned patterns. Detailed crystal structures of single CdS nanorods were characterized by HRTEM, as shown in Fig. 4. Examining many HRTEM images reveals the singlecrystalline nature of the nanorods, with surface roughness less than $0.5 \mathrm{~nm}$. The lattice spacing is identified to be $0.336 \mathrm{~nm}$, corresponding to the (002) plane of the wurzite CdS structure parallel to the long axis of the nanorods, which is the same as for the nanorods grown with $3 \mathrm{~nm}$ Au seeds. The inset in Fig. 4(a) is an FFT pattern from the larger CdS nanorod, which can be indexed as the [100] zone 
axis of the wurzite structure. In some of the samples, extended defects, most prominently stacking faults (SFs) can be observed at one end of the CdS nanorods as shown in Fig. 4(b), presumably resulting from the lattice mismatch between Au seeds and CdS. The energy-dispersive X-ray analysis (EDX) spectrum for the larger CdS nanorods shows the presence of $\mathrm{Cd}$ and $\mathrm{S}$ elements in the rods with an approximate molar ratio of 1.1:1.

The growth mechanism of the CdS nanorods is expected to be consistent with SLS. For SLS, typically the catalyst (Au NPs in this case) should be in a molten state. Although it is well known that the melting point of nanoparticles scales with size and a reduction of the bulk melting point of $1064{ }^{\circ} \mathrm{C}$ for $\mathrm{Au}$ of up to a few hundred degrees can be expected [31],

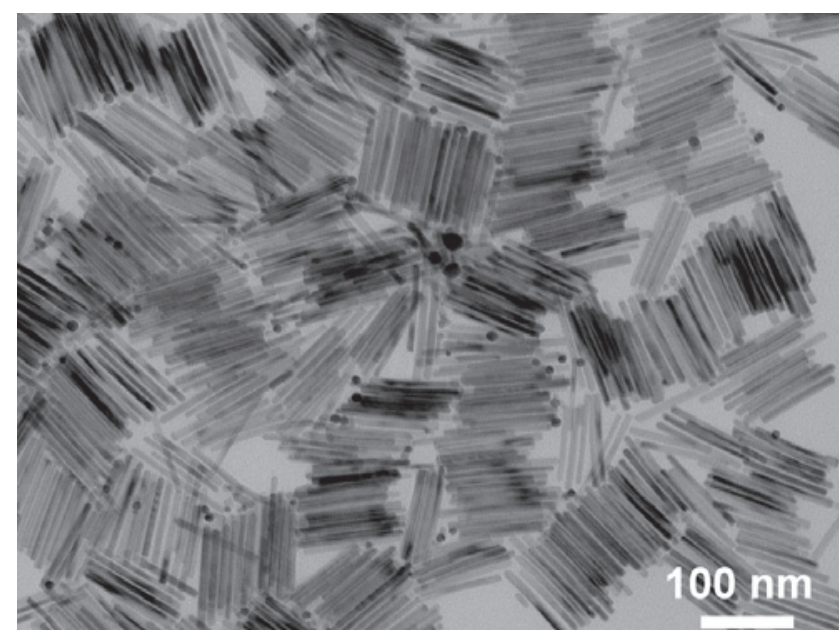

Figure 3 TEM image showing that the CdS nanorods prepared by anisotropic growth using $10 \mathrm{~nm}$ seed particles are very uniform, with a typical diameter of $10 \mathrm{~nm}$ and length about $120 \mathrm{~nm}$

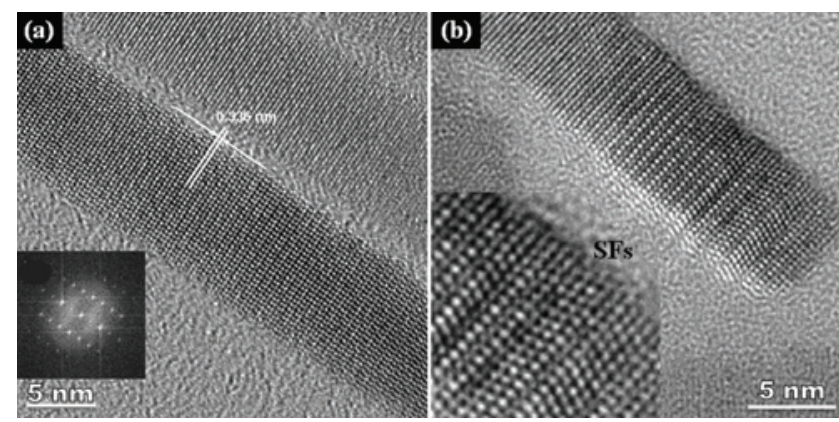

Figure 4 HRTEM images of CdS nanorods prepared by anisotropic growth on $10 \mathrm{~nm}$ seed particles. The lattice fringe of $0.336 \mathrm{~nm}$ in (a) corresponds to the (002) plane of the wurzite $\mathrm{CdS}$. The insert fast Fourier transform (FFT) pattern can be indexed as [100] of wurzite CdS. Stacking faults (SFs) can be observed at one end of the CdS nanorods (b) this still far exceeds the temperature $\left(300{ }^{\circ} \mathrm{C}\right)$ used in the reaction. Therefore it is unlikely that the whole nanoparticle catalyst melts. However, the surface melting temperature of NPs can be significantly lower than their interior due to the reduced number of cohesive bonds [32]. The quasi-molten surface layers will enhance the dissolution of the precursor materials and precipitation of solid crystals to form nanorods upon supersaturation. Meanwhile, since only the surface layer is mobile, the underlying symmetry of the crystal lattice of $\mathrm{Au}$ is preserved, which guides the heteroepitaxial growth of CdS nanorods. The growth of the rod-like geometry is a result of the crystal structure of wurzite CdS, which has strong tendency to grow along the closestpacked [001] direction. This is confirmed by careful examination of many HRTEM images.

HRTEM investigations on a small fraction of nanorods with Au seeds still attached can provide detailed information on the orientation relationship between $\mathrm{Au}$ particles and CdS nanorods. The most frequently observed $\mathrm{Au} / \mathrm{CdS}$ interfaces are shown by the HRTEM images in Fig. 5, in which several types of heteroepitaxial relationship can be observed. Figure 5(a) shows that the CdS nanorod grows along the [001] direction on the (111) plane of an Au seed. SFs can be clearly seen near the Au/CdS interface. A straightforward relationship of planar epitaxy can be proposed for the $\mathrm{CdS}(001)$ and $\mathrm{Au}(111)$ planes, in which, $10<100>_{\mathrm{CdS}}(0.4136 \mathrm{~nm})$ and $7<110>_{\mathrm{Au}}(0.5768$ $\mathrm{nm})$ are coincident with a $2.4 \%$ mismatch. In contrast, in Figs. 5(b) and 5(c), the (001) plane of CdS is parallel to the $\mathrm{Au}(200)$ and (110) planes, respectively. The conditions for the epitaxial relationships $\mathrm{Au}(200) / \mathrm{CdS}(001)$ and $\mathrm{Au}(110) / \mathrm{CdS}(001)$ can be stated in terms of continuity of atomic planes at the interfaces. For both conditions, the CdS(2-10) plane coincides with the $\mathrm{Au}(002)$ plane with a $\sim 1.4 \%$ misfit. However, there cannot be one-on-one lattice matching in the perpendicular direction. Instead, a regular correspondence between the two lattices can be envisioned as being every eight adjacent CdS(100) planes coinciding with seven $\mathrm{Au}(010)$ planes in (b), and ten $\mathrm{Au}(110)$ planes in (c). Occasionally, CdS nanorods were also found to grow on a highindex Au planes (not shown here). Schematics of the 


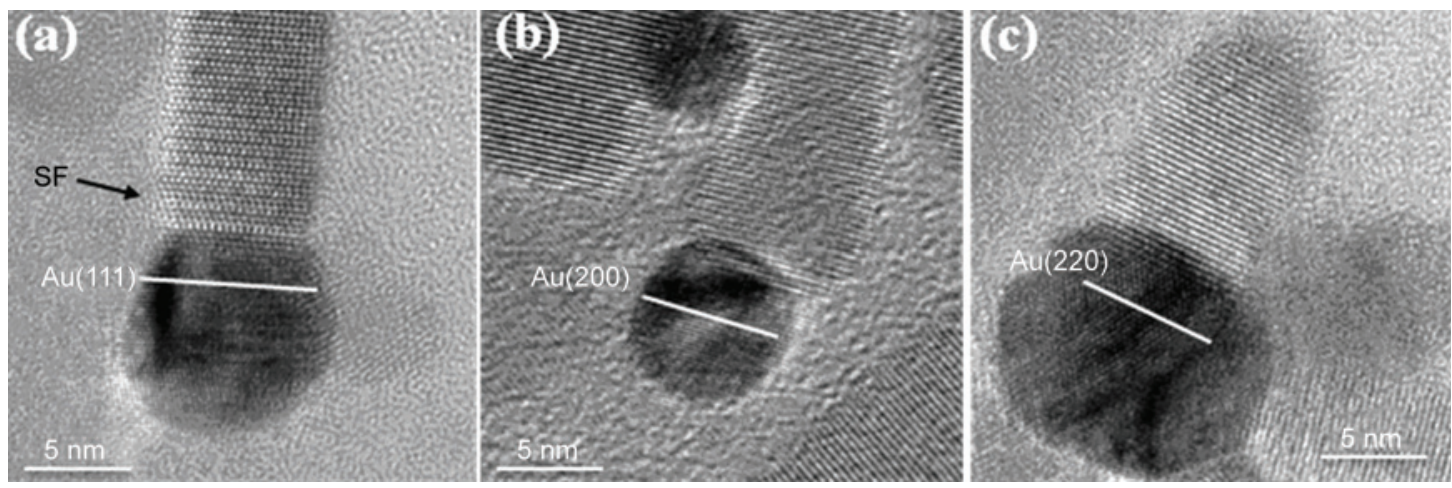

Figure 5 HRTEM images of the CdS-Au nanorods. The white lines represent the planes of the Au fcc structure. The relative orientations of the interfacial plane between the two domains are (a) Au(111)//CdS (001), (b) $\mathrm{Au}(200) / / \mathrm{CdS}(001)$, and (c) $\mathrm{Au}(220) / / \mathrm{CdS}(001)$

detailed heteroepitaxial relationship at the interfaces for all the three commonly observed situations are shown in the Electronic Supplementary Material (ESM) (Fig. S-1). It is likely that the epitaxial interfaces emerge from the crystallization of the amorphous CdS shell grown at low temperatures. As the temperature is raised, Au NP surfaces become molten and start to precipitate more CdS. The nanorods grow in length by pushing the initially nucleated material away from the Au seeds, during which the epitaxial relationship is retained. As will be discussed below, such epitaxial growth is critical for achieving uniform length of the nanorods.

The extremely uniform length of the nanorods is probably the result of the competition between the interface strain energy and bonding energy between the $\mathrm{Au}$ and $\mathrm{CdS}$. The lattice mismatch between $\mathrm{Au}$ and CdS causes strain between the two components. The total elastic strain energy scales with the length of the nanorod, while the chemical bonding energy is proportional to the interfacial area. As the aspect ratio increases, the elastic strain energy will eventually exceed bonding energy, and the rods will cleave from the surface of $\mathrm{Au}$ seeds and become free-standing nanorods. In the SLS mechanism, the precursor is fed from the catalyst side. Once the Au NP detaches from the nanorod, the CdS will cease growing. To confirm this proposed growth mechanism, two further experiments were performed: (1) In the final stage the synthesis temperature was kept at $300{ }^{\circ} \mathrm{C}$ for $40 \mathrm{~min}$. instead of $3 \mathrm{~h}$, and the reaction was subsequently quenched. The length of the CdS nanorods is found to be much shorter than $120 \mathrm{~nm}$ and varies widely, from 10-30 nm. All nanorods are still attached with $\mathrm{Au}$ NPs. This suggests that the nanorods do not grow at the same rate. However, they do grow to their full length as governed by the competing surface energetics as long as sufficient time is allowed. (2) In the final stage the synthesis temperature was kept at $300{ }^{\circ} \mathrm{C}$ for $12 \mathrm{~h}$. The nanorod morphology is the same as those heated for $3 \mathrm{~h}$, suggesting that once the nanorods detach from the Au catalysts, no further growth can occur. Therefore the length of the nanorod is governed by a self-limiting process.

Figure 6(a) shows the UV-vis absorption spectra of the smaller CdS-Au hybrid nanorods and larger CdS nanorods. The UV-vis spectrum of CdS-Au hybrid nanorods shows an absorption edge at $485 \mathrm{~nm}$ and that of the larger CdS nanorods appears at $490 \mathrm{~nm}$. Figure 6(b) shows the PL spectra of CdS-Au hybrid nanorods and larger CdS nanorods synthesized at $300{ }^{\circ} \mathrm{C}$. Compared with pure CdS quantum dots, the intensity of the PL peak (at $490 \mathrm{~nm}$ ) of the CdS-Au hybrid nanorods is significantly reduced. This is due to the intimate contact of the CdS nanorod with $\mathrm{Au}$, facilitating the transfer of photo-generated electrons from the semiconductor to the metallic component, and reducing the charge recombination. This phenomenon is consistent with previous reports for hybrid CdS-Au nanostructures [27]. The PL spectrum of the larger CdS nanorods shows a band edge emission at $495 \mathrm{~nm}$ along with a much stronger and broader low-energy peak centered at $750 \mathrm{~nm}$. The latter is believed to result from the trap-state emission arising from surface defect sites. These optical measurements are consistent with previous work and 


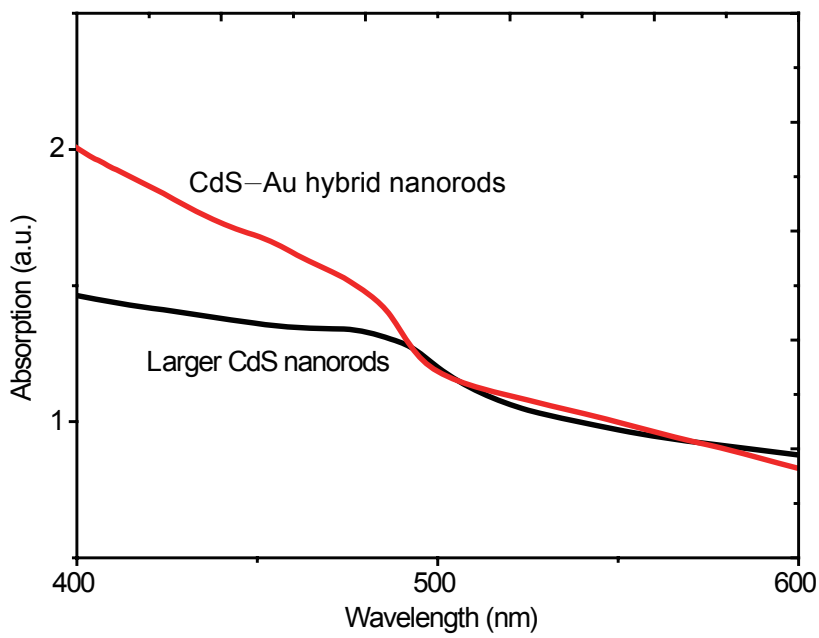

(a)

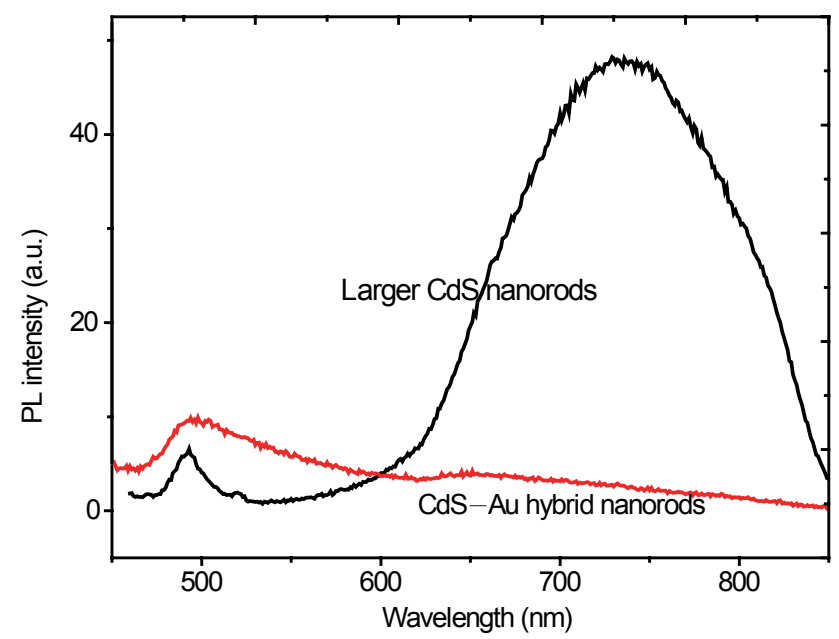

(b)

Figure 6 (a) UV-vis absorption spectra of CdS-Au hybrid nanorods and larger CdS nanorods; (b) PL emission spectra of larger CdS nanorods and CdS-Au hybrid nanorods

both the absorption and band edge emission spectra are blue-shifted from the bulk band gap value of CdS at $517 \mathrm{~nm}$ due to the quantum confinement in the transverse direction [28].

\section{Conclusions}

In summary, we report the solution phase synthesis of CdS nanorods catalyzed by Au nanoparticle seeds. Smaller $3 \mathrm{~nm}$ seeds lead to CdS-Au hybrid nanorods and larger $10 \mathrm{~nm}$ seeds result in monodispersed free-standing CdS nanorods. The nanorods grow primarily along the [001] axis of the wurzite structure. The monodispersity, with extremely uniform length and diameter, is governed by the competition between surface bonding energy and strain energy during the SLS growth. Both nanostructures show absorption that is blue-shifted slightly from the bulk CdS band-gap. A weak PL emission is observed for CdS-Au hybrid nanorods, whilst larger CdS nanorods show a strong and broad surface defect PL emission.

\section{Acknowledgments}

This work is supported by NSF-DMR 0547036, NSFCBET 0652042, and UB Integrated Nanostructured Systems Instrument Facilities.

Electronic Supplementary Material: Supplementary material is available in the online version of this article at http://dx.doi.org/10.1007/ s12274-008-8032-5 and is accessible free of charge.

\section{References}

[1] Alivisatos, A. P. Semiconductor clusters, nanocrystals, and quantum dots. Science 1996, 271, 933-937.

[2] Hu, J. T.; Odom, T. W.; Lieber, C. M. Chemistry and physics in one dimension: Synthesis and properties of nanowires and nanotubes. Acc. Chem. Res. 1999, 32, 435-445.

[3] Cozzoli, P. D.; Pellegrino, T.; Manna, L. Synthesis, properties and perspectives of hybrid nanocrystal structures. Chem. Soc. Rev. 2006, 35, 1195-1208.

[4] Murphy, C. J.; Jana, N. R. Controlling the aspect ratio of inorganic nanorods and nanowires. Adv. Mater. 2002, $14,80-82$

[5] Peng, X. G.; Manna, L.; Yang, W. D.; Wickham, J.; Scher, E.; Kadavanich, A.; Alivisatos, A. P. Shape control of CdSe nanocrystals. Nature 2000, 404, 59-61.

[6] Manna, L.; Milliron, D. J.; Meisel, A.; Scher, E. C.; Alivisatos, A. P. Controlled growth of tetrapod-branched inorganic nanocrystals. Nat. Mater. 2003, 2, 382-385.

[7] Manna, L.; Scher, E. C.; Alivisatos, A. P. Synthesis of soluble and processable rod-, arrow-, teardrop-, and tetrapod-shaped CdSe nanocrystals. J. Am. Chem. Soc. 2000, 122, 12700-12706.

[8] Trentler, T. J.; Goel, S. C.; Hickman, K. M.; Viano, A. M.; Chiang, M. Y.; Beatty, A. M.; Gibbons, P. C.; Buhro, W. E. Solution-liquid-solid growth of indium phosphide fibers from organometallic precursors: Elucidation of molecular and nonmolecular components of the pathway. J. Am. 
Chem. Soc. 1997, 119, 2172-2181.

[9] Trentler, T. J.; Hickman, K. M.; Goel, S. C.; Viano, A. M.; Gibbons, P. C.; Buhro, W. E. Solution-liquid-solid growth of crystalline III-V semiconductors - an analogy to vaporliquid-solid growth. Science 1995, 270, 1791-1794.

[10] Kan, S. H.; Aharoni, A.; Mokari, T.; Banin, U. Shape control of III-V semiconductor nanocrystals: Synthesis and properties of InAs quantum rods. Faraday Discuss. 2004, 125, 23-38.

[11] Hull, K. L.; Grebinski, J. W.; Kosel, T. H.; Kuno, M. Induced branching in confined PbSe nanowires. Chem. Mater. 2005, 17, 4416-4425.

[12] Shi, W. L.; Sahoo, Y.; Zeng, H.; Ding, Y.; Swihart, M. T.; Prasad, P. N. Anisotropic growth of PbSe nanocrystals on $\mathrm{Au}-\mathrm{Fe}_{3} \mathrm{O}_{4}$ hybrid nanoparticles. Adv. Mater. 2006, 18, 1889-1894.

[13] Grebinski, J. W.; Richter, K. L.; Zhang, J.; Kosel, T. H.; Kuno, M. Synthesis and characterization of Au/Bi core/ shell nanocrystals: A precursor toward II-VI nanowires. J. Phys. Chem. B 2004, 108, 9745-9751.

[14] Holmes, J. D.; Johnston, K. P.; Doty, R. C.; Korgel, B. A. Control of thickness and orientation of solution-grown silicon nanowires. Science 2000, 287, 1471-1473.

[15] Zhang, J. Z. Interfacial charge carrier dynamics of colloidal semiconductor nanoparticles. J. Phys. Chem. B 2000, 104, 7239-7253.

[16] Shen, G. Z.; Cho, J. H.; Yoo, J. K.; Yi, G. C.; Lee, C. J. Synthesis of single-crystal $\mathrm{CdS}$ microbelts using a modified thermal evaporation method and their photoluminescence. J. Phys. Chem. B 2005, 109, 9294-9298.

[17] Jang, J. S.; Joshi, U. A.; Lee, J. S. Solvothermal synthesis of CdS nanowires for photocatalytic hydrogen and electricity production. J. Phys. Chem. C 2007, 111, 13280-13287.

[18] Duan, X. F.; Niu, C. M.; Sahi, V.; Chen, J.; Parce, J. W.; Empedocles, S.; Goldman, J. L. High-performance thinfilm transistors using semiconductor nanowires and nanoribbons. Nature 2003, 425, 274-278.

[19] Weinhardt, L.; Gleim, T.; Fuchs, O.; Heske, C.; Umbach, E.; Bar, M.; Muffler, H. J.; Fischer, C. H.; Lux-Steiner, M. C.; Zubavichus, Y.; Niesen, T. P.; Karg, F. CdS and $\mathrm{Cd}(\mathrm{OH})_{2}$ formation during $\mathrm{Cd}$ treatments of $\mathrm{Cu}(\mathrm{In}, \mathrm{Ga})(\mathrm{S}, \mathrm{Se})_{2}$ thinfilm solar cell absorbers. Appl. Phys. Lett. 2003, 82, 571.

[20] Mandal, S.; Rautaray, D.; Sanyal, A.; Sastry, M. Synthesis and assembly of CdS nanoparticles in Keggin ion colloidal particles as templates. J. Phys. Chem. B 2004, 108, 7126-7131.

[21] Duan, X. F.; Lieber, C. M. General synthesis of compound semiconductor nanowires. Adv. Mater. 2000, 12, 298302.

[22] Ye, C. H.; Meng, G. W.; Wang, Y. H.; Jiang, Z.; Zhang, L. D. On the growth of CdS nanowires by the evaporation of CdS nanopowders. J. Phys. Chem. B 2002, 106, 10338-10341.

[23] Zhang, M. F.; Drechsler, M.; Muller, A. H. E. Templatecontrolled synthesis of wire-like cadmium sulfide nanoparticle assemblies within core-shell cylindrical polymer brushes. Chem. Mater. 2004, 16, 537-543.

[24] Xu, D.; Liu, Z. P.; Liang, J. B.; Qian, Y. T. Solvothermal synthesis of $C d S$ nanowires in a mixed solvent of ethylenediamine and dodecanethiol. J. Phys. Chem. B 2005, 109, 14344-14349.

[25] Carbone, L.; Nobile, C.; De Giorg, M.; Sala, F. D.; Morello, G.; Pompa, P.; Hytch, M.; Snoeck, E.; Fiore, A.; Franchini, I. R.; Nadasan, M.; Silvestre, A. F.; Chiodo, L.; Kudera, S.; Cingolani, R.; Krahne, R.; Manna, L. Synthesis and micrometer-scale assembly of colloidal CdSe/CdS nanorods prepared by a seeded growth approach. Nano Lett. 2007, 7, 2942-2950.

[26] Lin, H. Y.; Chen, Y. F.; Wu, J. G.; Wang, D. I.; Chen, C. C. Carrier transfer induced photoluminescence change in metal-semiconductor core-shell nanostructures. Appl. Phys. Lett. 2006, 88, 161911.

[27] Saunders, A. E.; Popov, I.; Banin, U. Synthesis of hybrid CdS-Au colloidal nanostructures. J. Phys. Chem. B 2006, 110, 25421-25429.

[28] Yong, K. T.; Sahoo, Y.; Swihart, M. T.; Prasad, P. N. Shape control of CdS nanocrystals in one-pot synthesis. J. Phys. Chem. C 2007, 111, 2447-2458.

[29] Brust, M.; Walker, M.; Bethell, D.; Schiffrin, D. J.; Whyman, R. Synthesis of thiol-derivatized gold nanoparticles in a 2-phase liquid-liquid system. J. Chem. Soc. Chem. Commun. 1994, 801-802.

[30] Yu, H.; Chen, M.; Rice, P. M.; Wang, S. X.; White, R. L.; Sun, S. H. Dumbbell-like bifunctional $\mathrm{Au}-\mathrm{Fe}_{3} \mathrm{O}_{4}$ nanoparticles. Nano Lett. 2005, 5, 379-382.

[31] Buffat, P.; Borel, J. P. Size effect on melting temperature of gold particles. Phys. Rev. A 1976, 13, 2287-2298.

[32] Sakai, H. Surface-induced melting of small particles. Surf. Sci. 1996, 351, 285-291. 